\title{
Atherosclerotic lesions and mitochondria DN A deletions in brain microvessels: Implication in the pathogenesis of A lzheimer's disease
}

\author{
Gjumrakch Aliev ${ }^{1}$ \\ Eldar Gasimov² \\ Mark E O brenovich ${ }^{3}$ \\ Kathryn Fischbach ${ }^{1}$ \\ Justin C Shenk ${ }^{1}$ \\ Mark A Smith ${ }^{3}$ \\ George Perry 3,4
}

${ }^{1} \mathrm{D}$ epartment of Biology and ${ }^{4} \mathrm{C}$ ollege of Sciences, U niversity of Texas at San Antonio, San Antonio, Texas, USA ; ${ }^{2}$ D epartment of $\mathrm{H}$ istology and Embryology, Baku Medical U niversity, Baku, A zerbaijan; ${ }^{3} D$ epartment of Pathology, C ase W estern Reserve U niversity, C leveland, O hio, USA
Correspondence: Gjumrakch Aliev Department of Biology, College of Sciences and Electron Microscopy Research Center, The U niversity of Texas at San Antonio, 0 ne UTSA Circle, San Antonio,TX 78249-1664, USA

$\mathrm{Tel}+12104584518$

Fax +12104584506

Email gjumrakch.aliev@ utsa.edu

\begin{abstract}
The pathogenesis that is primarily responsible for Alzheimer's disease (AD) and cerebrovascular accidents (CVA) appears to involve chronic hypoperfusion. We studied the ultrastructural features of vascular lesions and mitochondria in brain vascular wall cells from human $\mathrm{AD}$ biopsy samples and two transgenic mouse models of $\mathrm{AD}$, yeast artificial chromosome (YAC) and C57B6/SJL Tg (+), which overexpress human amyloid beta precursor protein $(\mathrm{A} \beta \mathrm{PP})$. In situ hybridization using probes for normal and $5 \mathrm{~kb}$ deleted human and mouse mitochondrial DNA (mtDNA) was performed along with immunocytochemistry using antibodies against the $\mathrm{A} \beta$ peptide processed from $\mathrm{A} \beta \mathrm{PP}, 8$-hydroxy-2'-guanosine $(8 \mathrm{OHG})$, and cytochrome c oxidase (COX). More amyloid deposition, oxidative stress markers as well as mitochondrial DNA deletions and structural abnormalities were present in the vascular walls of the human AD samples and the AßPP-YAC and C57B6/SJL Tg (+) transgenic mice compared to age-matched controls. Ultrastructural damage in perivascular cells highly correlated with endothelial lesions in all samples. Therefore, pharmacological interventions, directed at correcting the chronic hypoperfusion state, may change the natural course of the development of dementing neurodegeneration.
\end{abstract}

Keywords: atherosclerosis, Alzheimer's disease, transgenic animals, brain hypoperfusion, vascular and mitochondrial lesions, electron microscopy

\section{Introduction}

Vascular endothelial cells, neurons and glia are all able to synthesize, store and release reactive oxygen species (ROS) and vasoactive substances in response to certain stimuli, especially those produced by chronic hypoxia/hypoperfusion (for review see Aliev et al 2003b). The contribution of these substances to the pathophysiology of stroke, cerebrovascular disease or CVAs and AD is extremely important. It has been suggested that hypoperfusion can be an initiator of AD (Aliev et al 2002b, 2003a, 2003b, 2007; de la Torre 2002a). This idea is based on a positive correlation between $\mathrm{AD}$ and cardiovascular diseases. ROS are generated at sites of injury and/or inflammation. We hypothesize that the cellular and molecular mechanisms, by which hypoperfusion-induced ROS-accumulation impairs endothelial barrier function and promotes leukocyte adhesion, induce alterations in normal vascular function and result in the development of AD (Aliev et al 2003b).

Sustained hypoperfusion promoting oxidative stress of brain tissues could also stimulate secondary damage via the overexpression of inducible and neuronal specific nitric oxide synthase (iNOS and nNOS, respectively) and endothelin-1 (ET-1) in brain cells (Aliev et al 2000; de la Torre 2002a; Aliyev et al 2004; de la Torre and Aliev 2005). The continuous accumulation of oxidative stress products, such as peroxynitrite accumulation (via the overexpression of the iNOS and/or nNOS), may be secondary as well as an accelerating factor for the damage and compromise of the 
blood brain barrier (BBB) in hypoxia/hypoperfusion or $\mathrm{AD}$ (Aliyev et al 2004).

One of the main effects of chronic hypoperfusion induced vascular abnormality in $\mathrm{AD}$ appears to be tissue oxygen deficiency. Chronic cerebral hypoperfusion induces reduction of tissue oxygen delivery that causes the development of cognitive impairments such as AD (Kumar et al 1990; Friston and Frackowiak 1991; De Jong et al 1997; de la Torre 1997, 2002a; Aliev et al 2007). De la Torre (2000) proposes that advanced aging with a comorbid condition, such as a vascular risk factor that further decreases cerebral perfusion, promotes a critically attained threshold of cerebral hypoperfusion (CATCH). With time, $\mathrm{CATCH}$ induces brain capillary degeneration and suboptimal delivery of energy substrates to neuronal tissue (de la Torre 2000). Because glucose is the main fuel of brain cells, its impaired delivery together with a deficient delivery of oxygen, compromises neuronal stability because the supplies for aerobic glycolysis fail to meet the brain tissue demand. The outcome of CATCH is a metabolic cascade that involves, among other things, mitochondrial dysfunction, oxidative stress, decreased adenosine triphosphate (ATP) production and increased calcium entry, abnormal protein synthesis, cell ionic pump deficiency, signal transduction defects, and neurotransmission failure. These events contribute to the progressive cognitive decline characteristic of patients with $\mathrm{AD}$, as well as regional anatomic pathology consisting of synaptic loss, senile plaques (SP), neurofibrillary tangles (NFT), tissue atrophy, and neurodegeneration. $\mathrm{CATCH}$ encompasses the clinical heterogenic pattern that characterizes $\mathrm{AD}$ and provides compelling evidence that any of a multitude of different etiopathophysiologic vascular risk factors, in the presence of advanced aging, can lead to AD (de la Torre 2000, 2002b; de la Torre and Aliev 2005; Aliev et al 2007).

The aim of the present study was to investigate the features of the vascular lesions in human $\mathrm{AD}$ and two transgenic mouse models of $\mathrm{AD}$, lines overexpressing a mutated form of the amyloid beta precursor protein (A $\beta P P)$. We compared the ultrastructural features of each and identified common changes relative to age-matched controls that may give clues to underlying mechanisms affecting brain neuronal function.

\section{Materials and methods}

All experimental procedures were performed in accordance with University of Texas San Antonio and Case Western Reserve University guidelines for the use of human biopsy and post-mortem tissues and the care and use of laboratory animals for research. Human AD brain biopsy and postmortem tissues for ultrastructural studies were taken as described earlier and processed for future electron microscopic (EM) and in situ hybridization studies (Hirai et al 2001; Aliev et al 2002a, 2002b, 2003a, 2007). Mouse brain samples were obtained from a normal, nontransgenic line (Py8.9-Hemi [wild type]) and 2 transgenic lines, AßPP-YAC and C57B6/SJL Tg (+), were obtained from Bruce Lamb and colleagues (1999). Animals were 22-26 months old ( $n=6$ for each group). All animals received a standard laboratory diet ad libitum. Mice, under terminal anesthesia were perfusion fixed via the heart as described previously (Aliev et al 2002a, 2003a; Aliyev et al 2005). Brain samples were processed for future analysis by electron microscope and in situ hybridization for cytological detection of mtDNA on the electron microscopic level. In situ hybridization was performed using wild type and $\Delta 5 \mathrm{~kb}$ deleted human and mouse specific probes as described recently (Aliev et al 2003a; Aliev 2007). Finally, all the sections were exposed to $\mathrm{OsO}_{4}$ for $1 \mathrm{~h}$ at RT, rinsed, dehydrated and flat embedded in Spurr's embedding media. Ultrathin sections were stained with uranylacetate and lead citrate and viewed in a JEOL 100CX or Jeol 1230 EX electron microscope at $80 \mathrm{kV}$.

\section{Results and discussion}

Endothelial cells (EC) and perivascular cells of human brain microvessels from age-matched control cases showed no visible changes in their ultrastructure. Mitochondria in the EC were intact (data not shown).

Conversely, cortical microvessels from $\mathrm{AD}$ brain biopsies were characterized by different degrees of damage, and heterogeneous lesions (Figures 1A-C). In some areas microvessels showed multiple lesions such as the presence of a cluster of mitochondria-derived lysosomes and necrotic changes in the ultrastructure of the vascular EC and perivascular cells (Figures 1A-B). Very often capillary endothelium cells showed the presence of "giant" sized lipid vacuoles in their matrices. Transformation of the mitochondria to mitochondria-derived lysosomes was generalized to all brain cellular compartments (Figures 1B-C). In addition, EC occupied only a small part of the vessel wall. Perivascular cells showed the presence of a large number of the mitochondria-derived vacuoles in their matrices (Figure 1C). Sometimes microvascular endothelium, at the early stages of $\mathrm{AD}$, did not show any damage in their ultrastructure. However, the luminal plasma membrane of this EC sharply protruded into the vessel lumen, indicating the effect of hypoperfusion before any visible ultrastructural damage (Figure 1A). 

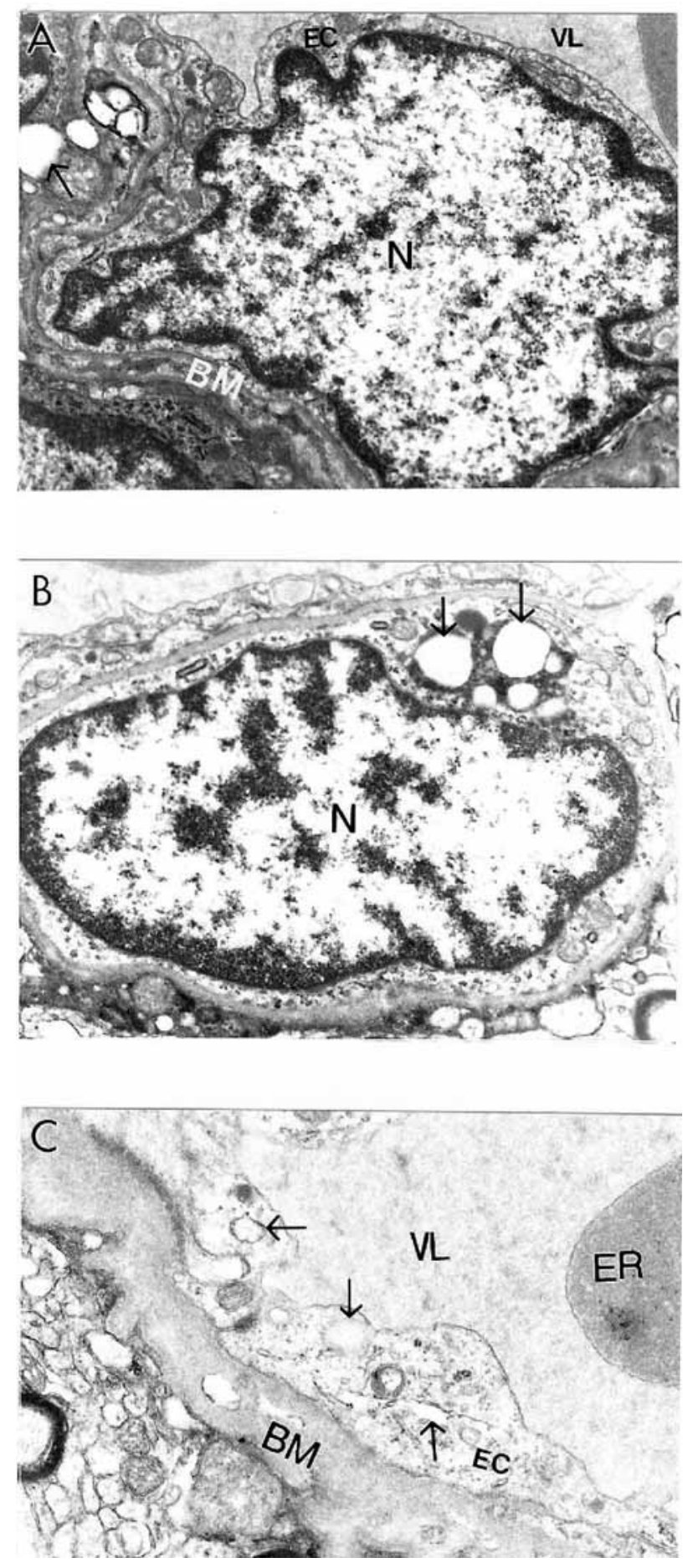

Figure $1 \mathrm{U}$ Itrastructural characteristics of brain microvessels from human A Izheimer disease brain biopsies. A: Microvessels with minimal changes did not show any particular pathology in the ultrastructure of the vascular endothelium; however, perivascular cells show the presence of vacuolar degenerative structures in the matrix (indicated by single arrow).B:Vascular endothelium and perivascular cells of damaged microvessels display ultrastructural lesions in their cytoplasmic organelles especially mitochondria. Completely damaged matrices appeared to be permanent features of all of these cells (indicated by single arrow). 0 riginal magnification $\times 20,000$ (A and B). C: Vascular endothelium with non-reversible damage have completely damaged mitochondria and destructive changes in the membranous structures. The basal membrane (BM) is very thick and occupies a large area of the vascular wall. 0 riginal magnification $\times 12,000$.
The ultrastructural features of vascular lesions and mitochondria changes in neuronal cell bodies in transgenic and non-transgenic age-matched control mice were analyzed following perfusion fixation (Aliev et al 2000, 2003a, 2005). Ultrastructural changes in the brain samples of A $\beta$ PP-YAC, but not age-matched control mice, coexisted with different degrees of amyloid deposition (Aliev 2002; Aliev et al 2002a).

Very often, clusters of $A \beta$ positive immunoreactivity were observed in the neuronal cell bodies of perivascular paired cortical neurons (Aliev et al 2000; Aliev 2002). EM immunocytochemistry revealed different sizes of fibrils and extracellular types of amyloid deposits in the brain tissues (Figure 2A-F). The amyloid depositions were associated with the formation of parietal helical filamental (PHF) structures (Figure 2D-F), which is a permanent feature of neuronal lesions in AD brains (Aliev et al 2000, 2003a).

Brain microvessels showed immunopositive staining for $\mathrm{A} \beta$ and were characterized by the presence of large-sized lipid-laden vacuoles in the matrices of EC and perivascular cells (Figure 3A-D). These changes were generalized to cortical microvessels as in AD samples (Aliev 2002). The ultrastructural abnormalities of vascular wall cells were dependent on the presence of $A \beta$ deposits around the microvessels (Figures 3-4). In contrast with these observations, age-matched control mouse brain vessels did not show any particular changes in the ultrastructure of vascular EC at different levels of microcirculation. Only sometimes did a very small number of lipid droplets appear in the matrices of perivascular cells (Aliev 2002; Aliev et al 2003a). This data clearly indicates that disruption of BBB function in vascular EC may be a major factor in lipid accumulation and amyloid deposition during the development of AD-like pathology in AßPP-YAC mice without cholesterol feeding (Aliev 2002; Shi et al 2002b; Aliev et al 2003a).

Ultrastructural immunocytochemical analyses using peroxidase-anti-peroxidase (PAP) and/or colloidal gold probes indicated that the damaged vascular walls in A $\beta P P-Y A C$ transgenic mice possess atherosclerotic lesions (Figure 4A-B), while control and non-damaged vessels from A $\beta P P-Y A C$ mice do not show $A \beta$ immunopositive staining (data not shown).

Age-matched control mice showed no particular changes in their neuronal ultrastructure (Figure 5A-B). Cortical neuronal cell bodies in A $\beta$ PP-YAC mice were characterized by different degrees of ultrastructural alterations in their mitochondrial structures (Figure 6A-F) similar to human AD samples (Aliev et al 1999, 2000). Giant and electrondense (ED) mitochondria appeared to be permanent features 

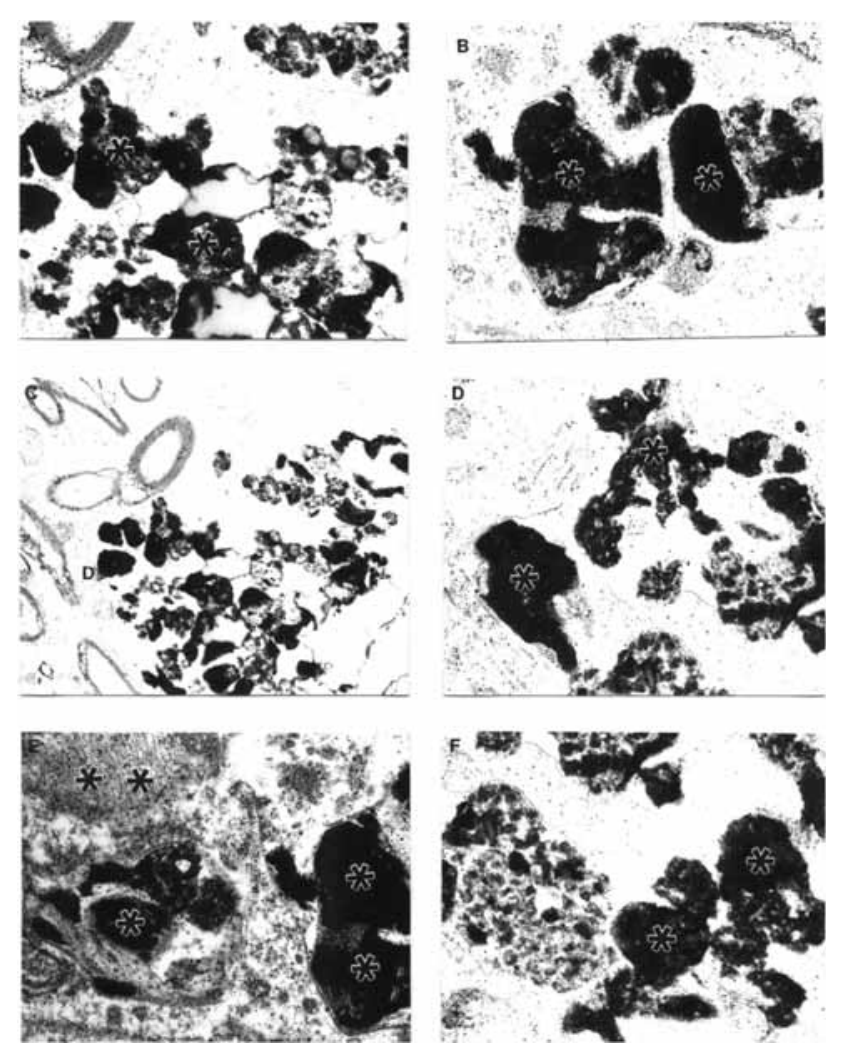

Figure 2 Features of amyloid deposition in a neuronal cell body and the extracellular matrix in A $\beta P P$-YAC aged transgenic mouse brain (PAP pre-embedding immunocytochemistry). Amyloid depositions are associated with the formation of PHF-like structures (double asterisk in figure $\mathbf{E}$ ). 0 riginal magnification $\mathbf{A - D} \times 20,000$. E-F $\times 25,000$.

of the neuronal abnormality and was associated with the A $\beta$ PP overexpression (Aliev et al 2000, 2003a). We also found that mitochondrial lesions in neuronal cell bodies appeared to be associated with the absence of microtubules and with lipofuscin formation. The same observations were seen in the populations of vulnerable neurons in $\mathrm{AD}$ brain biopsies indicating neuronal cytoskeletal abnormalities (Cash et al 2003).

We looked at ultrastructural features of brain tissue from C57B6/SJL Tg (+) transgenic mice overexpressing AßPP. We demonstrated that the binding of basic fibroblast growth factor (bFGF) and serum amyloid $\mathrm{P}$ (SAP) to $\mathrm{A} \beta$ is a marker of a lack of BBB integrity in this mouse model. Adjacent sections of brain were stained with $4 \mathrm{G} 8$, a monoclonal antibody to the $\mathrm{A} \beta$ peptide (aa17-24), followed by 48.1 , a monoclonal antibody against $b F G F$. The binding of bFGF in this mouse line is similar to that of $\mathrm{AD}$ cases in which bFGF binds specifically to $A \beta$ neuritic plaques and the basement membrane of cerebral microvessels (Shi et al 1999, 2002a). In addition, the cores of amyloid plaques were intensely stained with $4 \mathrm{G} 8$, and bFGF binding is co-localized with amyloid immunoreactivity as visualized by polyclonal antiserum to amyloid. Our ultrastructural study indicated that bFGF immunostaining in aged C57B6/ SJL Tg (+) mice was associated with damaged, but not normal neurons (Aliev et al 2000, 2002a, 2003a, 2007). Moreover, this was associated with different degrees of $A \beta$ immunostaining in the neuronal cell body and vascular wall cells (Aliev 2002; Aliev et al 2003a; Aliyev et al 2005). The degree of mitochondrial abnormality, such as ED mitochondria-derived lysosomes and lipofuscin formation appeared to be features of damaged neurons in 24-month old aged C57B6/SJL Tg (+) but not age-matched control mice (Figure 7A-D). Vascular abnormality was associated with the selective damage to cortical neurons suggesting a direct relationship between vascular abnormality, BBB breakdown, neuronal loss and amyloid depositions during the maturation of AD-like pathology (Aliev 2002; Aliev et al 2003a).

Previously we demonstrated that the ultrastructural features of cortical neurons from AD brain biopsies are characterized by the selective localization of mitochondria abnormalities to the cell bodies (Aliev et al 1999, 2000; Hirai et al 2001; Aliev 2002; Shi et al 2002b). The majority of the neurons, which were closely associated with lesioned vessels, possessed different degrees of ultrastructural abnormality. Partially and completely damaged mitochondria were associated with lipofuscin formation and mitochondria appeared to be a major substrate for this process (Figures 6-7). In many cases, neuronal cell bodies showed an absence of cellular
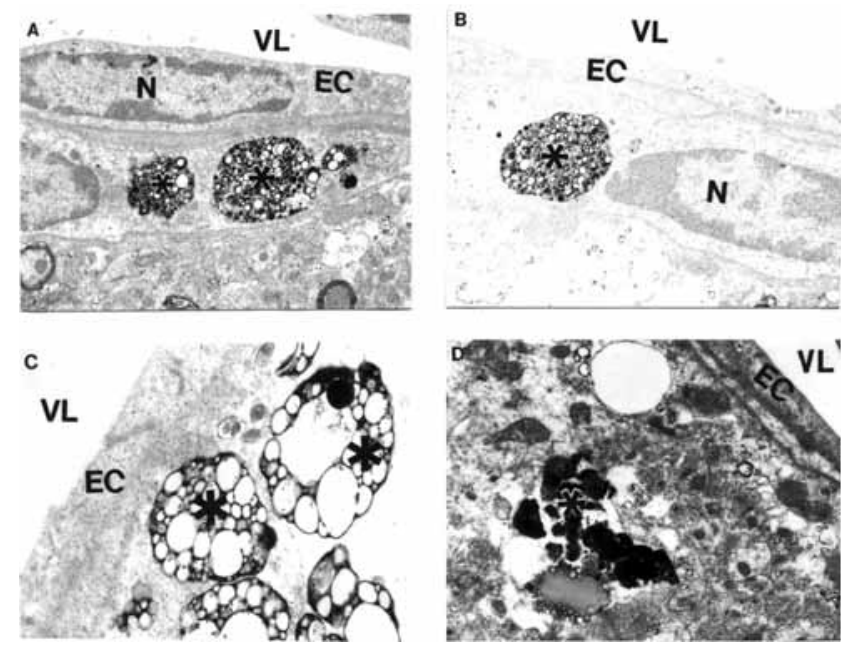

Figure 3 Features of APP immunostaining in frontal cortical microvessels in aged A $\beta$ PP-YAC mice determined by PAP pre-embedding immunocytochemistry. A, B and $\mathbf{C}$ are without counter staining. $\mathbf{D}$ is with counter staining. Asterisks indicate amyloid deposition. 0 riginal magnification $\times 12,000$. 

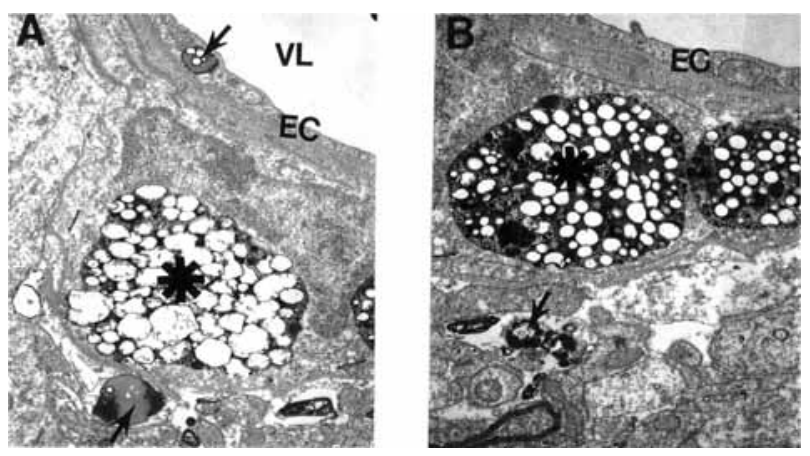

Figure 4 Electron microscopic subcellular features of brain cortical microvessels from 24-month old A $\beta$ PP-YAC mice. A: Vascular endothelial cells display lipid vacuoles in their cytoplasmic matrices (single arrow). Perivascular cells have "giant"- sized lipid/amyloid granules in their cytoplasmic matrices (indicated by asterisk). 0 riginal magnification: $\times 12,000$. B:Microvessels with amyloid "angio pathy" are characterized by the accumulation of large-sized amyloid/lipid granules in the matrices of perivascular cells (indicated by asterisk).Vacuolar structures with lipid filled amyloid deposition (single arrow) are also present in the matrices of perivascular cells. 0 riginal magnification: $\times 12,000$.

organelles. Different stages of mitochondrial abnormality, such as formation of mitochondria-derived lysosomes and lipofuscin, were seen in damaged but not in normal neurons (Figures 6-7). The mitochondria-derived lysosomes and lipofuscin deposits of varied density and sizes were permanent features of the neuronal abnormality (Aliev et al 2003a). Mitochondrial lesions and lipofuscinogenesis were also present in glial cells of the brain parenchyma (Aliev et al 2003a).

Quantitative morphometric measurements of the percentage of the different types of mitochondria (normal, partially damaged, and completely damaged) indicate that agematched control groups have a significantly higher percentage of normal mitochondria compared with AD cases (Hirai et al 2001). Cytological in situ hybridization studies using probes for human normal and $5 \mathrm{kB}$ deleted mtDNA found that mtDNA signals were associated with severely damaged or mitochondria-derived lysosomal structures (Figure 8). However, the areas containing lipofuscin did not show any mtDNA containing positive signals. On the ultrastructural level, clusters of $5 \mathrm{~kb}$ deleted mtDNA containing gold particles $(17 \mathrm{~nm})$ were localized in mitochondria-derived lysosomal structures, indicating that mtDNA deletions and turnover occur at the late stages of the mitochondrial lesion formation. Similarly, areas of neuronal cell bodies containing normal mtDNA and lipofuscin were free from any $5 \mathrm{~kb}$ deleted mtDNA positive signals (Hirai et al 2001; Aliev et al 2002a). In contrast to this observation, hippocampal neuronal mitochondria of age-matched control subjects did not show mtDNA positive signals containing gold particles in their matrices.
Our detailed immunocytochemical analysis also demonstrated that the mitochondrial abnormalities in neurons are associated with increased markers of lipid peroxidation (Moreira et al 2007). Moreover, lipid peroxidation markers were associated with RNA oxidation (staining by $8 \mathrm{OHG}$ ). Clusters of $8 \mathrm{OHG}$ containing immunopositive gold particles $(17 \mathrm{~nm})$ were localized to the matrices of completely and/or partially damaged mitochondria (Nunomura et al 2001; Aliev et al 2002, 2002a). Large quantities of immunopositive gold particles were also associated with the cytoplasmic matrix. In addition, the cytoplasmic matrices, especially of the regions where damaged mitochondria or mitochondria-lipofuscin were present, showed clusters of $8 \mathrm{OHG}$ containing immunopositive gold particles (Aliev et al 2002, 2002a). Our quantitative study indicated that the extent of oxidative damage (eg, $8 \mathrm{OHG}$ staining) is highly dependent on the degree of mitochondrial abnormalities (Nunomura et al 2001; Aliev et al 2003a).
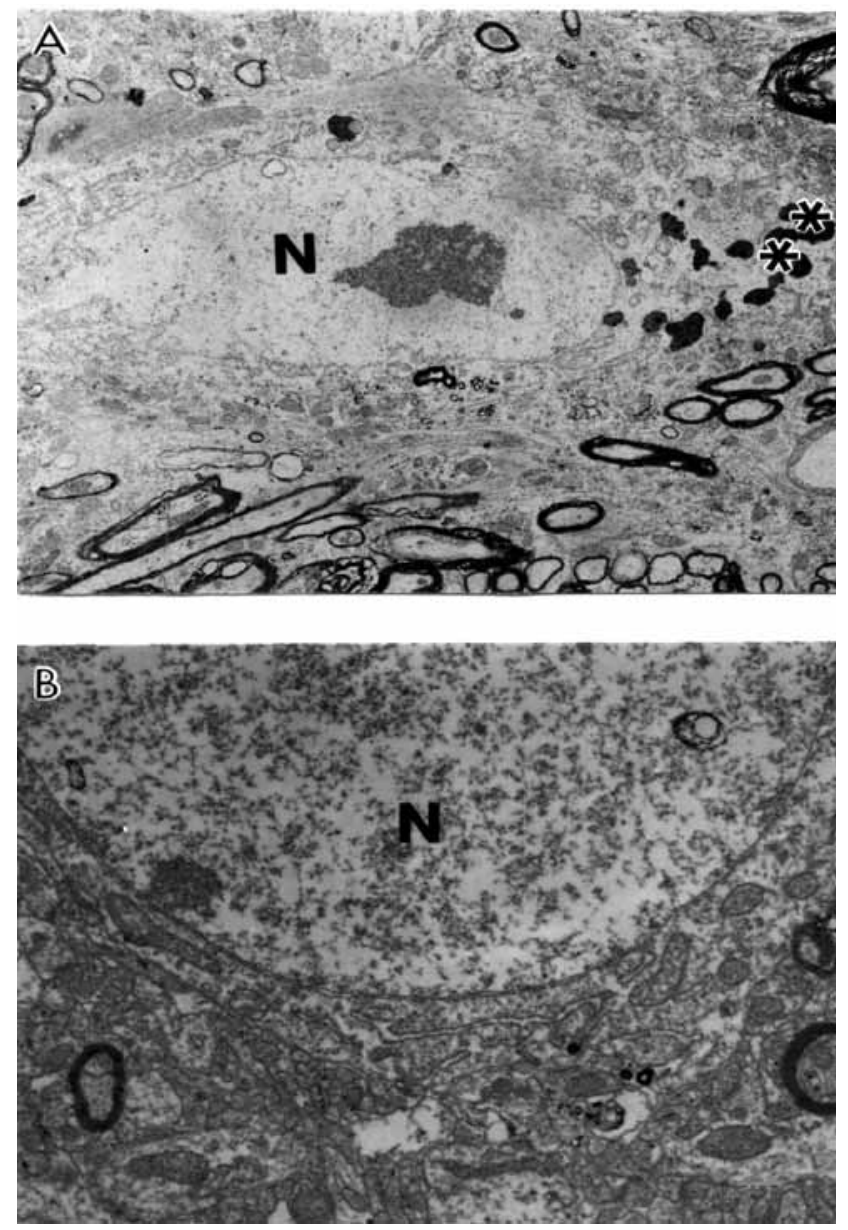

Figure 5 Age-matched control, non-transgenic mice did not show any particular changes in their neuronal ultrastructure. Lipofuscin was present in some neurons (indicate by asterisk in figure $\mathbf{A}) .0$ riginal magnification of $\mathbf{A}$ and $\mathbf{B}: \times 5,000$, and $\times 20,000$, respectively. 

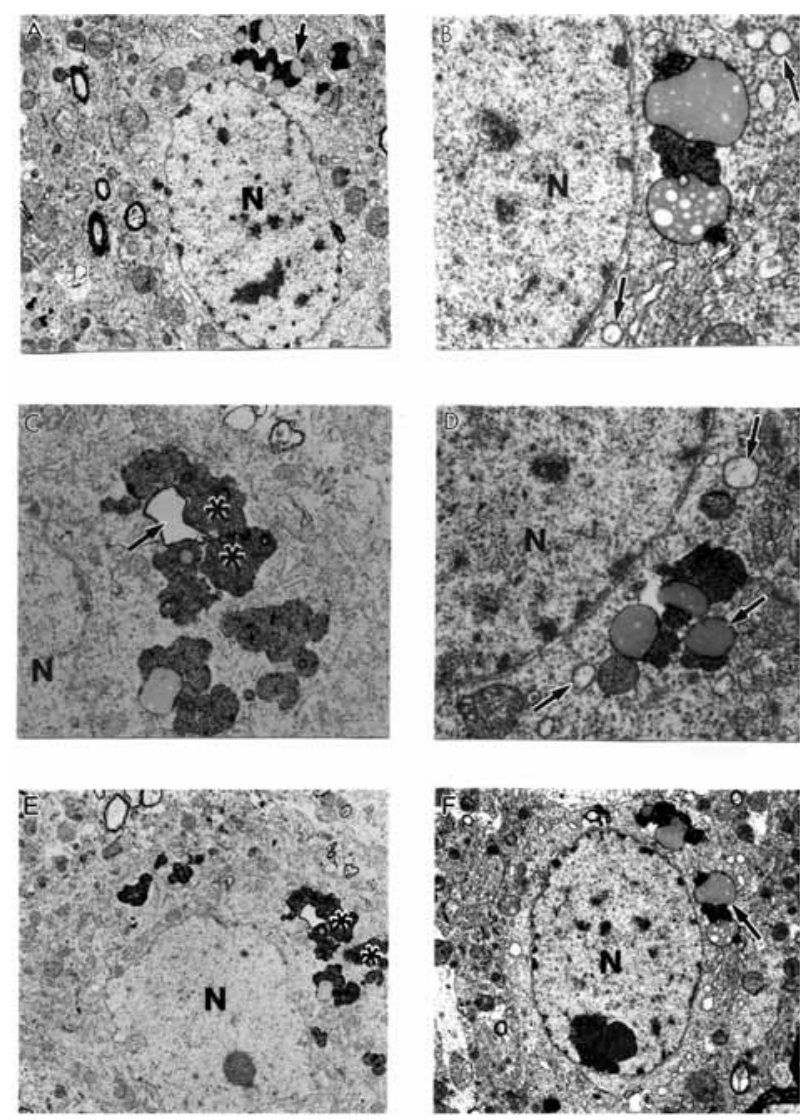

Figure 6 Ultrastructural characteristics of the neuronal damage in A $\beta P P-Y A C$ transgenic mouse hippocampus. Mitochondrial lesions were associated with lipofuscin formation in the neuronal cell body (asterisk) and mitochondria appeared to be a major substrate for lipofuscin formation (single arrows). 0 riginal magnification: $\mathbf{A}, \mathbf{E}$, and $\mathbf{F} \times 5,000$. B, $\mathbf{C}$, and $\mathbf{D} \times 20,000$ respectively.

Based on the evidence presented in this study we theorize the hypothetical timeline of the damage in the brain during maturation of $\mathrm{AD}$ is much more complex than the previously proposed hypothetical schematic (Aliev et al 2003b). With the onset of $\mathrm{AD}$, normal neurons develop numerous forms of oxidative damage including nitration (nitrotyrosine), lipid peroxide adducts (lipid peroxidation), and nucleic acid oxidation prior to the formation of pre-neurofibrillary tangles (pre-NFT) that induce nonreversible damage to neurons and therefore finally failure of neurotransmission that occurs in $\mathrm{AD}$ brain.

The detailed analysis of $8 \mathrm{OHG}$ immunostaining demonstrated that only vulnerable neurons show immunopositive staining for $8 \mathrm{OHG}$ in $\mathrm{AD}$, but not in age-matched controls. By using ultrastructural analysis we have found that $8 \mathrm{OHG}$ immunostaining was selectively associated in vulnerable neurons and microvessels of AD brain (Aliev et al 2002b; Aliyev et al 2005). The 8 OHG immunogold labeling (17 nm) was seen throughout the cytoplasm, including the damaged mitochondria or electron dense abnormal mitochondria (Hirai et al 2001; Nunomura et al 2001; Aliev et al 2002b; Moreira et al 2007). However, we did not find $8 \mathrm{OHG}$ in normal mitochondria or in lipofuscin. The capillary EC and perivascular pericytes showed a high intensity of $8 \mathrm{OHG}$ immunostaining (Aliev et al 2002b).

In this study, the main location of the mtDNA was in damaged mitochondria and mitochondria derived lysosomes, but not in lipofuscin in all brain cellular compartments including the vascular wall cells (Figure 8A-B). Endothelial cells (EC) of vessels with atherosclerotic lesions and nearby perivascular cells contain clusters of normal and deleted mtDNA positive signals (Figure $8 \mathrm{~A}-\mathrm{B}$ ). These observations emphasize the key role of hypoperfusion, mitochondrial abnormality and oxidative stress in the pathogenesis of vascular and nonvascular cells lesions during the development of AD-like pathology in AßPP-YAC mice (Aliev et al 2002a; Aliyev et al 2005). Many of these characteristics overlap the neuropathology of human AD (Aliev et al 2002a).

In situ hybridization analysis with mouse mtDNA probes found abundant deleted mtDNA in AßPP-YAC mice compared to age-matched controls (Aliev et al 2000, 2002a, 2003a). Moreover, the majority of deleted mtDNA was found in mitochondria-derived lysosomes (Figure 8C-D) in regions closely associated with lipofuscin, suggesting that proliferation, deletion and duplication of mtDNA occur in mitochondria, many of which have been fused with lysosomes (Aliev et al 2000). These finding suggest that abnormalities in mitochondrial structures and mtDNA are features of damaged neurons in A $\beta$ PP-YAC mice, and may also play a key role in the pathogenesis of AD (Aliev et al 2000). We found that neurons in AD samples were dominated by abnormal mitochondria as compared with the control group (Aliyev et al 2005). By in situ hybridization analyses with a chimeric cDNA probe to the $5 \mathrm{~kb}$ common deletion (Hirai et al 2001), we found that deleted mtDNA is increased at least 3 fold for the AD cases compared to the controls (Hirai et al 2001). In situ hybridization detected mtDNA proliferation, deletion and duplication in abnormal mitochondria, many of which were fused with lysosomes, indicating they were being turned over (see Figure 8A-B).

A growing body of evidence suggests that there are direct links between heart disease, hypertension, stroke and neurodegeneration (Roher et al 2003; de la Torre 2006; Skoog and Gustafson 2006). The relationship between atherosclerosis, vascular amyloidosis and brain hypoperfusion in the pathogenesis of sporadic Alzheimer's disease has been well 

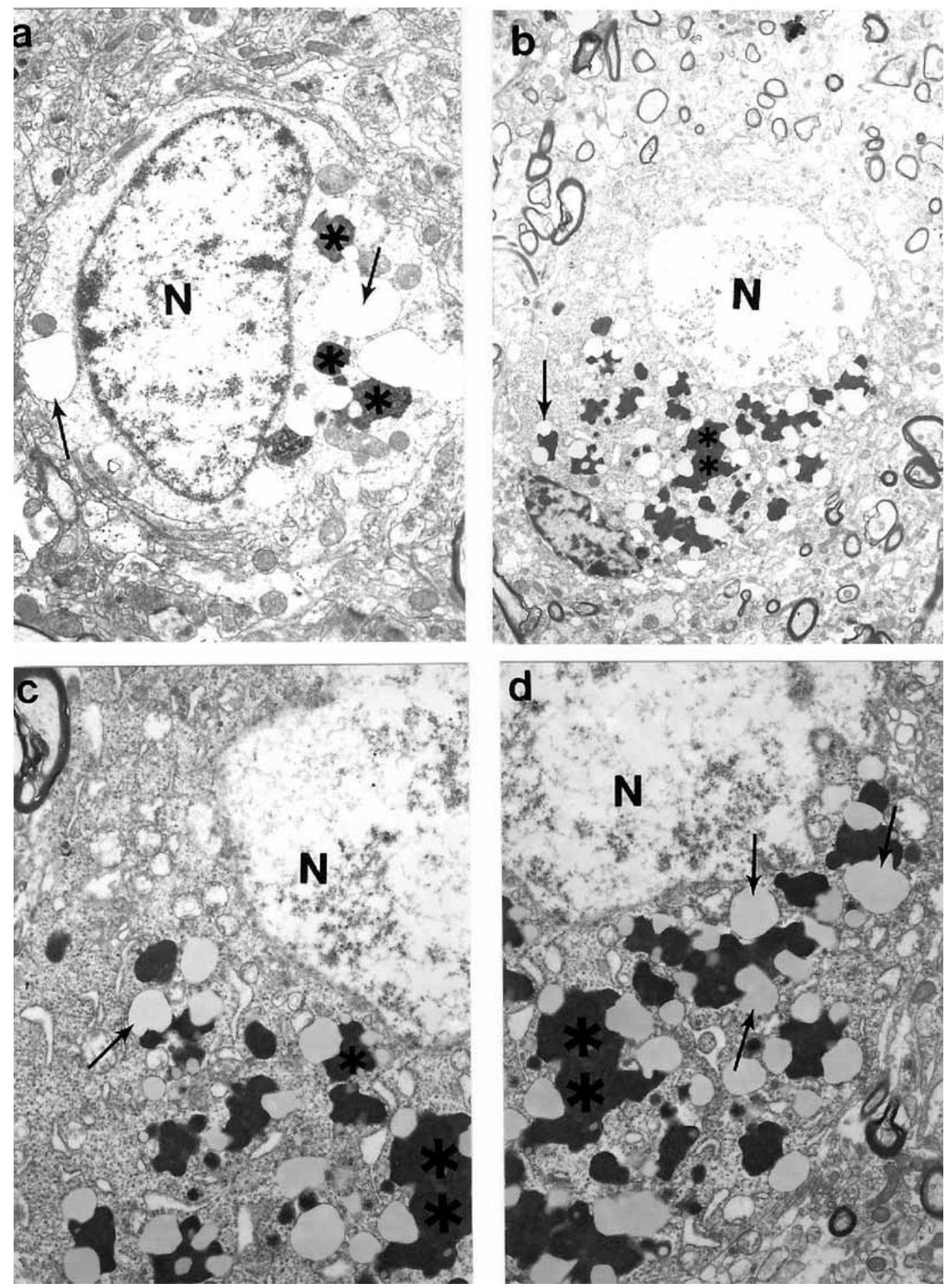

Figure $7 \mathrm{U}$ Itrastructural characteristics of cortical neurons in C 57B6/SJL transgenic mouse $(\mathrm{Tg}+)$ brain. The main characteristic of neuronal damage appeared to be the transformation of completely damaged mitochondria (mitochondria without any residues of mitochondrial cristae; indicated by single arrows) into the lipofuscin granules, characterised by their cluster type localization in the neuronal cell body. 0 riginal magnification: $\mathbf{A}$ and $\mathbf{B} \times 5,000 ; \mathbf{C}$ and $\mathbf{D} \times 20,000$, respectively.

documented (Roher et al 2003; Kalback et al 2004). Hypertension has been suggested to be an initiator of the ischemic pathway leading to AD (Aliev et al 2003, 2007; de la Torre 2006; Skoog et al 2006). Recently, studies by Choi and colleagues (2007) showed that preconditioning with chronic cerebral hypoperfusion reduces a focal cerebral ischemic injury and increases apurinic/apyrimidinic endonuclease/ redox factor-1 and matrix metalloproteinase-2 expression. Another report by Annaházi and colleagues (2007) demonstrated that the pre- and post-treatment with alpha-tocopherol 

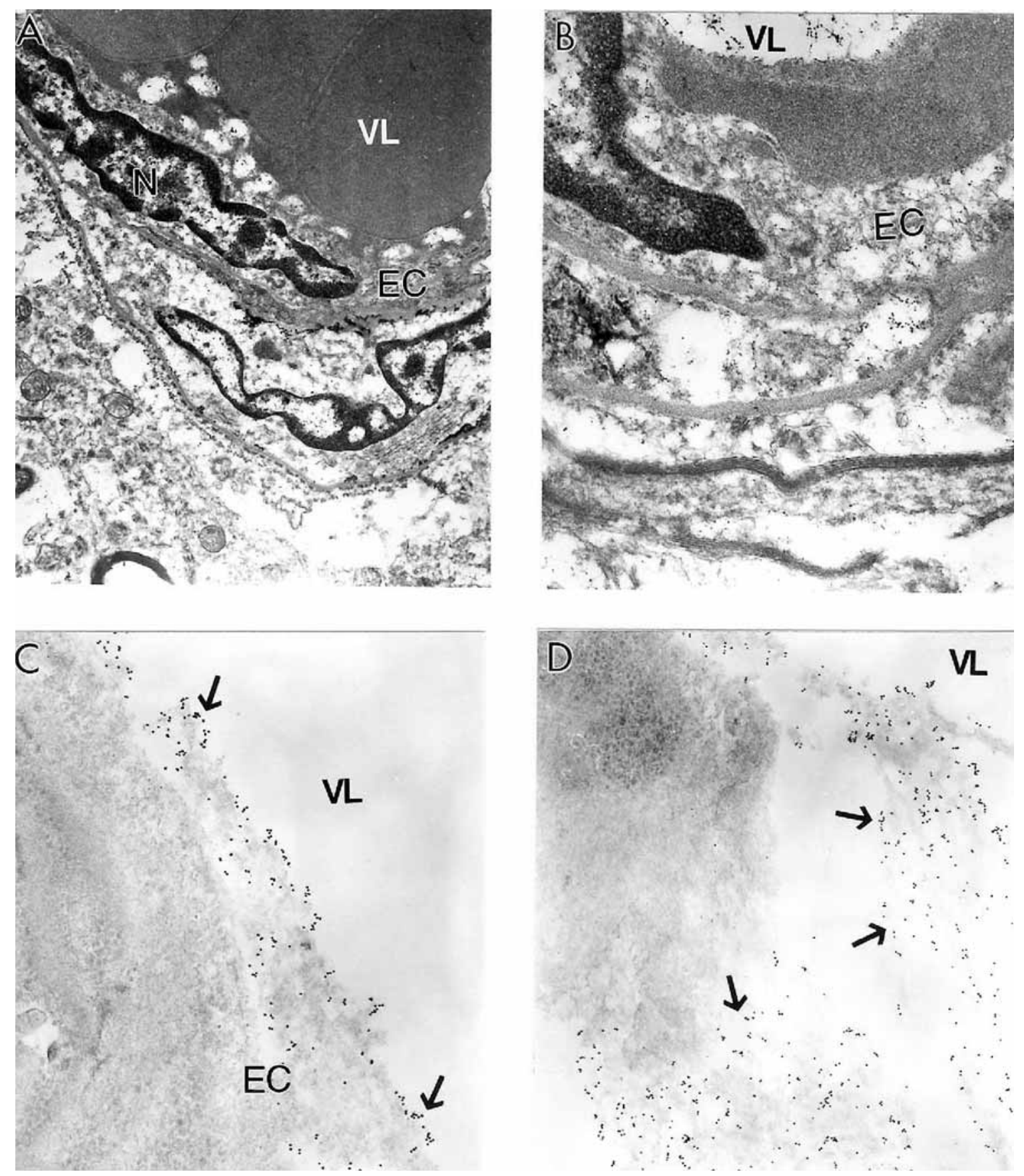

Figure 8 Electron microscopic determination of mitochondrial DN A signals visualized by using wild type and chimeric $5 \mathrm{~kb}$ deleted mtD N probes in a human postmortem Alzheimer's disease (AD) brain (A-B) and 24-month old A BPP-YAC transgenic mouse brain (C-D). A and B:AD brain microvessels endothelium and perivascular cells show clusters of wild type mtD N A containing positive signals visualized by indirect colloidal gold techniques (indicated by the dark dots). 0 riginal magnification: $\mathbf{A}$ and $\mathbf{B} \times 10,000$ and $\times 25,000$, respectively. C-D:A $\beta P P$-YAC transgenic mice show the presence of clusters of chimeric $5 \mathrm{~kb}$ deleted mitochondria D N A positive signals throughout the matrices of vascular and perivascular cells (single arrows). 0 riginal magnification: $\mathbf{C}$ and $\mathbf{D} \times 30,000$ and $\times 20,000$, respectively.

Abbreviations: BM, basal membrane; EC, endothelial cell; ER, erythrocytes; $\mathrm{N}$, cell nucleus; VL, vessel lumen; PA P, peroxidase-anti-peroxidase; PHF, paired helical filaments.

attenuates hippocampal neuronal damage in experimental cerebral hypoperfusion. Moreover, circulating CD34 positive T-cells are able to provide an index of cerebrovascular function (Taguchi et al 2004). The beneficial effect of carotid endarterectomy on the cerebral blood flow and cerebral blood volume improvement was studied by SPECT (Lushmanov et al 1997).

Based on this literature evidence and our present study we believed that managing $\mathrm{AD}$ as a vascular disease will open a new window of insight not only for better understanding of 
the etiopathogenesis but also new and more effective treatment strategies.

We theorize that the oxidative stress markers seen in the human $\mathrm{AD}$ and $\mathrm{AD}$ mouse models selectively affect the population of vulnerable neurons, vascular EC and perivascular cells. We believe that hypoperfusion-induced oxidative stress plays a key role in the pathogenesis of vascular and nonvascular cell lesions during the maturation of AD.

\section{Conclusion}

Our data for the first time demonstrates that a chronic injury stimulus induces the hypoperfusion seen in the microcirculation of vulnerable brain regions. Determining the mechanisms behind these imbalances may provide crucial information in the development of new, more effective therapies for the treatment of cerebrovascular diseases, including AD. Future studies must seek to answer the following questions: (1) What are the major factors altering and/or controlling cerebral blood flow during the progression of chronic hypoperfusion and/or the development of atherosclerotic changes in brain microvessels? (2) What are the roles of endothelial content vasoactive substances (especially nitric oxide [NO] and endothelin-1 [ET-1]) during the development of this pathophysiology? (3) Does acute or chronic hypoperfusion with concomitant oxidative stress accelerate the vascular and neuronal lesions during normal aging and/or when brain is exposed to chronic hypoperfusion induced factors? Resolving these issues will allow for novel therapeutic approaches that will modify the natural history of these chronic age onset disorders in the 21 st century.

\section{Acknowledgments}

Supported by the Philip Morris USA Research Management Group and Alzheimer's Association. The authors report no conflict of interest.

\section{References}

Aliev G. 2002. Is non-genetic Alzheimer's disease a vascular disorder with neurodegenerative consequences? J Alzheimer's Dis, 4:513-16.

Aliev G, Liu J, Xu K, et al. 2007. ApoE4 in mice induces age-dependent brain hypoperfusion, neuronal, glial and microvascular damage, and cognitive impairment, which can be prevented by feeding Acetyl-LCarnitine and R-Lipoic Acid. In: 8th International Conference on Alzheimer's and Parkinson's Diseases AD/PD (Salzburg, Austria). Proceeding AD/PD Conference 2007. Monduzzi Editore, Bologna, Italy. 2007 in press.

Aliev G, Obrenovich ME, Seyidova D, et al. 2005. Exploring ischemiainduced vascular lesions and potential pharmacological intervention strategies. Histol Histopathol, 20:261-73.

Aliev G, Seyidova D, Lamb BT, et al. 2003a. Mitochondria and vascular lesions as a central target for the development of Alzheimer's disease and Alzheimer disease-like pathology in transgenic mice. Neurol Res, 25:665-74.
Aliev G, Seyidova D, Neal ML, et al. 2002a. Atherosclerotic lesions and mitochondria DNA deletions in brain microvessels as a central target for the development of human $\mathrm{AD}$ and AD-like pathology in aged transgenic mice. Ann N Y Acad Sci, 977:45-64.

Aliev G, Shi J, Perry G, et al. 2000. Neuronal mitochondrial abnormalities in yeast artificial chromosome (YAC) transgenic mice overexpressing amyloid precursor protein (APP). Society for Neuroscience Abstracts Book, 30.

Aliev G, Smith MA, Obrenovich ME, et al. 2003b. Role of vascular hypoperfusion-induced oxidative stress and mitochondria failure in the pathogenesis of Alzheimer disease. Neurotox Res, 5:491-504.

Aliev G, Smith MA, Seyidov D, et al. 2002b. The role of oxidative stress in the pathophysiology of cerebrovascular lesions in Alzheimer's disease. Brain Pathol, 12:21-35.

Aliev G, Smith MA, Vinters H, et al. 1999. Mitochondria abnormalities mark vulnerable neurons in Alzheimer's disease. J Neuropathol Exp. Neurol, 58:511.

Aliyev A, Chen SG, Seyidova D, et al. 2005. Mitochondria DNA deletions in atherosclerotic hypoperfused brain microvessels as a primary target for the development of Alzheimer's disease. J Neurol Sci, 229-230:285-92.

Aliyev A, Seyidova D, Rzayev N, et al. 2004. Is nitric oxide a key target in the pathogenesis of brain lesions during the development of Alzheimer's disease? Neurol Res, 26:547-53.

Annaházi A, Mracskó E, Süle Z, et al. 2007. Pre-treatment and posttreatment with alpha-tocopherol attenuates hippocampal neuronal damage in experimental cerebral hypoperfusion. Eur J Pharmacol, 571(2-3):120-8

Cash AD, Aliev G, Siedlak SL, et al. 2003. Microtubule reduction in Alzheimer's disease and aging is independent of tau filament formation. Am J Pathol, 162:1623-7.

Choi SA, Kim EH, Lee JY, et al. 2007. Preconditioning with chronic cerebral hypoperfusion reduces a focal cerebral ischemic injury and increases apurinic/apyrimidinic endonuc-lease/redox factor-1 and matrix metalloproteinase-2 expression. Curr Neurovasc Res, 4:89-97.

De Jong GI, De Vos RA, Steur EN, et al. 1997. Cerebrovascular hypoperfusion: a risk factor for Alzheimer's disease? Animal model and postmortem human studies. Ann N Y Acad Sci, 826:56-74.

de la Torre JC. 1997. Hemodynamic consequences of deformed microvessels in the brain in Alzheimer's disease. Ann N Y Acad Sci, 826:75-91.

de la Torre JC. 2000. Critically attained threshold of cerebral hypoperfusion: the CATCH hypothesis of Alzheimer's pathogenesis. Neurobiol Aging, 21:331-42.

de la Torre JC. 2002a. Alzheimer's disease: how does it start? J Alzheimer's Dis, 4:497-512.

de la Torre JC. 2002b. Alzheimer disease as a vascular disorder: nosological evidence. Stroke, 33:1152-62.

de la Torre JC. 2006. How do heart disease and stroke become risk factors for Alzheimer's disease? Neurol Res, 6:637-44.

de la Torre JC, Aliev G. 2005. Inhibition of vascular nitric oxide after rat chronic brain hypoperfusion: spatial memory and immunocytochemical changes. J Cereb Blood Flow Metab, 25:663-72.

Friston KJ, Frackowiak RS. 1991. Cerebral function in aging and Alzheimer's disease: the role of PET. Electroencephalogr Clin Neurophysiol Suppl, 42:355-65.

Hirai K, Aliev G, Nunomura A, et al. 2001. Mitochondrial abnormalities in Alzheimer's disease. J Neurosci, 21:3017-23.

Lamb BA, Bardel KA, Kulnane LS, et al. 1999. Amyloid production and deposition in mutant amyloid precursor protein and presenilin-1 yeast artificial chromosome transgenic mice. Nat Neurosci, 2:695-7.

Kumar A, Schapiro MB, Haxby JV, et al. 1990. Cerebral metabolic and cognitive studies in dementia with frontal lobe behavioral features. J Psychiatr Res, 24:97-109.

Kalback W, Esh C, Castaño EM, et al. 2004. Atherosclerosis, vascular amyloidosis and brain hypoperfusion in the pathogenesis of sporadic Alzheimer's disease. Neurol Res, 26:525-39. 
Lishmanov Y, Shvera I, Ussov W, et al. 1997. The effect of carotid endarterectomy on cerebral blood flow and cerebral blood volume studied by SPECT. J Neuroradiol, 24:155-62.

Moreira PI, Siedlak SL, Wang X, et al. 2007. Autophagocytosis of mitochondria is prominent in Alzheimer disease. J Neuropathol Exp Neurol, 66:525-32.

Nunomura A, Perry G, Aliev G, et al. 2001. Oxidative damage is the earliest event in Alzheimer disease. J Neuropathol Exp Neurol, 60:759-67.

Roher AE, Esh C, Kokjohn TA, et al. 2003. Circle of willis atherosclerosis is a risk factor for sporadic Alzheimer's disease. Arterioscler Thromb Vasc Biol, 23:2055-62.

Shi J, Perry G, Aliev G, et al. 1999. Serum amyloid P is not present in amyloid beta deposits of a transgenic animal model. Neuro Report, 10:3229-32.
Shi J, Perry G, Berridge MS, et al. 2002a. Labeling of cerebral amyloid beta deposits in vivo using intranasal basic fibroblast growth factor and serum amyloid $\mathrm{P}$ component in mice. $\mathrm{J} \mathrm{Nucl} \mathrm{Med,}$ 43:1044-51.

Shi J, Seyidova D, Perry G, et al. 2002b. Neuronal mitochondria abnormalities in a transgenic mouse model overexpressing amyloid beta. Society for Neuroscience 295.20.

Skoog I, Gustafson D. 2006. Update on hypertension and Alzheimer's disease. Neurol Res, 28:605-11.

Taguchi A, Matsuyama T, Moriwaki H, et al. 2004. Circulating CD34positive cells provide an index of cerebrovascular function. Circulation, 109:2972-5. 